\title{
A CASE OF UNILATERAL BULBAR LESION, PROBABLY SYRINGOBULBIA, WITH SPECIAL REFERENCE TO THE SENSORY PATHWAYS WITHIN THE MEDULLA.
}

\author{
By W. G. WYLLIE, London.
}

\section{INTRODUCTION.}

OF the descriptions available of dissociated sensory disturbances resulting from lesions of the medulla that which is associated with the occlusion of a posterior inferior cerebellar artery is by far the most frequently met with. The medullary syndrome effected by such a lesion is well known. On the other hand, the descriptions of similar disturbances in sensation consequent upon medullary lesions due to other causes are less numerous.

Clinical examination in the case to be described gave evidence of changes in sensation in many ways similar to those which occur in cases of occlusion of a posterior inferior cerebellar artery. Taking into account, however, the age of the patient, the sequence of the symptoms and their relatively slow accumulation, I am of the opinion that the lesion was not one of vascular origin. It is suggested, for reasons to be given later, that the case was one of syringobulbia.

Although handicapped by the want of a precise pathological definition of the nature and limits of the lesion in question, yet the character of the disturbances effected in the various sensory modalities was thought to be of sufficient interest for their publication. To Dr. Kinnier Wilson, under whose care the patient was admitted to the National Hospital, Queen Square, I wish here to express my indebtedness for permission to make the following report.

\section{DESCRIPTION OF CASE.}

Miss L. H., sixteen years old, draper's assistant, was admitted to hospital in December, 1922. At the age of ten she had had an operation for adenoids, and had suffered from 'rheumatism,' first at four years of age, and frequently since in the arms, shoulders and legs. Her father died of cancer at the age of forty-four; her mother was living. Of nine children only one brother and one sister survived besides herself; the remainder died in infancy. She stated that for a twelvemonth before admission her voice had become of a lower pitch. In the same period several deep cracks had developed in the skin of the fingers of the right 
hand. These were painless and very slow in healing, and she did not remember any injuries likely to have caused their appearance.

In November, 1922, she became unsteady on her legs. Shortly afterwards, in the same month, she began to experience a sensation of coldness in the right extremities and half of the body. She further observed that on placing the right arm in hot or cold water she was unable to appreciate the temperature of the water. Following upon the abnormality in sensation on the right side, a feeling of coldness developed in the left half of the face. Also the left pupil became smaller, and the left eyelid drooped slightly. She stated that she mistrusted her left hand, as she occasionally dropped things which she attempted to hold in it (this symptom subsequently disappeared). She had a tendency to be unsteady in walking, but was doubtful which leg was at fault. Swallowing was apt to make her cough. It seemed at times 'as if something pushed the food back into the mouth again.' Shortly before admission to hospital she received a small burn on the right hand, which produced a blister. This injury was not attended by any pain.

State on Admission.-The patient was of medium height, small-boned and poorly nourished. No abnormality was observed in the shape of the head. The teeth were well formed but asymmetrical, the left lower central incisor being almost in the mid-line. No deformity of the spine was noticed, except that the spinous processes of the ninth to

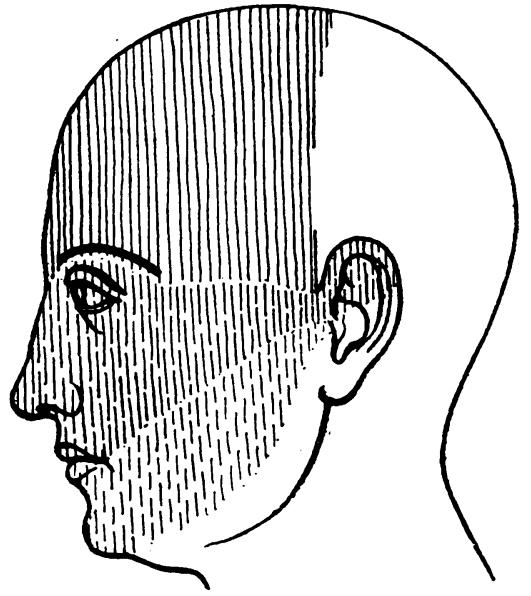

FIg. 1.-Showing variations in loss to painful cutaneous stimuli over left face.

the twelfth dorsal vertebræ were bifid at their extremities. The cardiovascular, renal, alimentary and respiratory systems were unaffected.

The Nervous System.- - The fundi and visual fields were normal. A reduction in the size of the left pupil and palpebral fissure was apparent. The pupil reactions and ocular movements were undisturbed, and no nystagmus or diplopia was present. The left corneal reflex was absent. In the distribution of the left trigeminal nerve minimal tactile stimuli were appreciated, but felt lighter than did similar stimuli upon the right half of the face. Pin-prick over the left fifth was felt as a blunt point upon the forehead, and as 'very dull' below this down to a line running roughly from the corner of the mouth to the tragus of the ear. Below this again, over the remainder of the fifth distribution, pin-prick was less dull (Fig. 1). Appreciation of heat was absent upon the forehead and 
showed similar variations below that level to those of painful sensibility. Cold was said to be sometimes warm, sometimes neutral, the perverse sensation being most frequent below the level of the forehead.

As regards sensation inside the mouth, pin-prick was reduced in appreciation on the left half of the tongue and on the left side of the soft palate, fauces and pharynx. The touch of a camel-hair brush could be felt on the left side of the tongue, palate, fauces and nasopharynx, but was said to be less than on the right side. There was a difference in

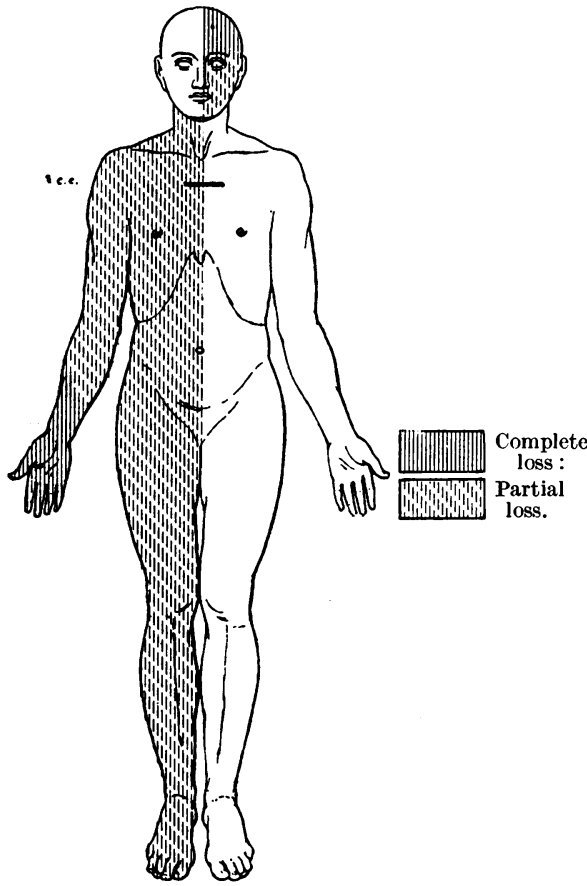

Fia. 2.-Analgesia.

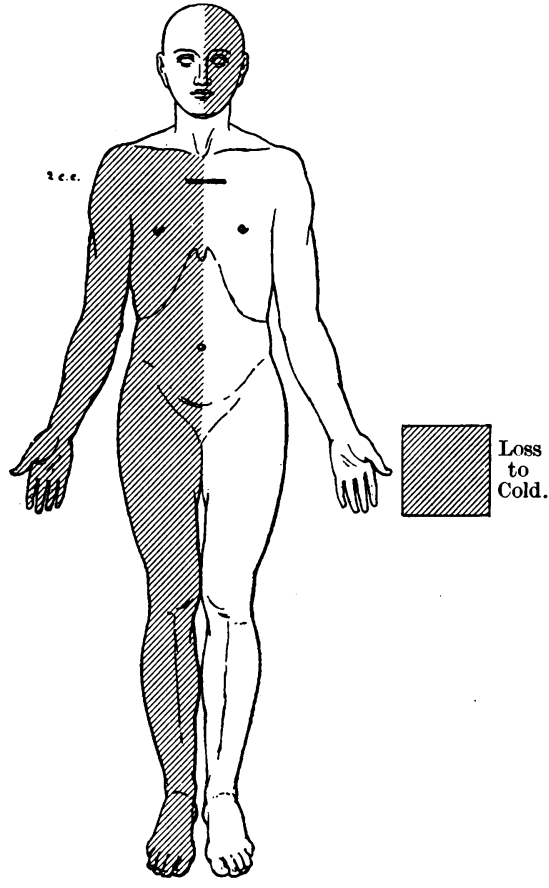

FIG. 3.

pressure pain upon the two sides of the tongue, sensibility being less upon the left than upon the right side.

The palatal and pharyngeal reflexes were absent, except for a very weak pharyngeal reflex upon the right side. On phonation the palate moved equally well upon both sides, but the left vocal cord was seen to be fixed in the abducted position. There was occasional difficulty in swallowing. The tongue on protrusion deviated slightly towards the left. Taste was unaffected on both halves of the tongue.

The integrity of the seventh, eighth and eleventh nerves was preserved upon the left side, and no defects were observed in the cranial nerves upon the right side beyond the absence of the palatal reflex already mentioned. 
Sensation on the right extremities and half of the body was severely disturbed, and less deeply upon the right side of the neck. Sensibility to heat, cold and pain was markedly reduced (Figs. 2, 3, 4). Very hot stimuli were observed as being ' just warm ' or ' warm.' More mistakes were made with cold, which was sometimes mistaken for warmth, and frequently said to be neutral. Tactile sensibility, however, was very lightly impaired upon the right side, in that cotton wool felt lighter than upon contralateral parts, but all stimuli were appreciated (Fig. 5).

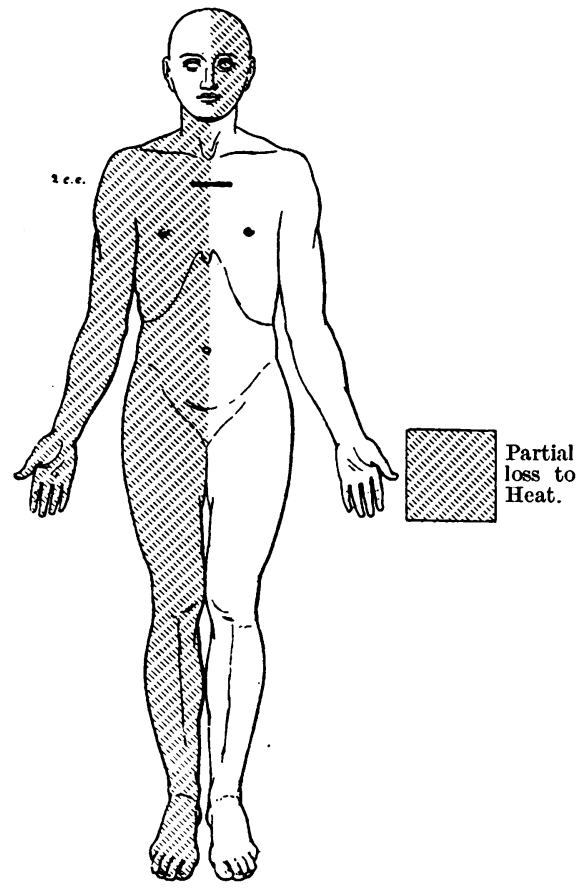

Fig. 4.

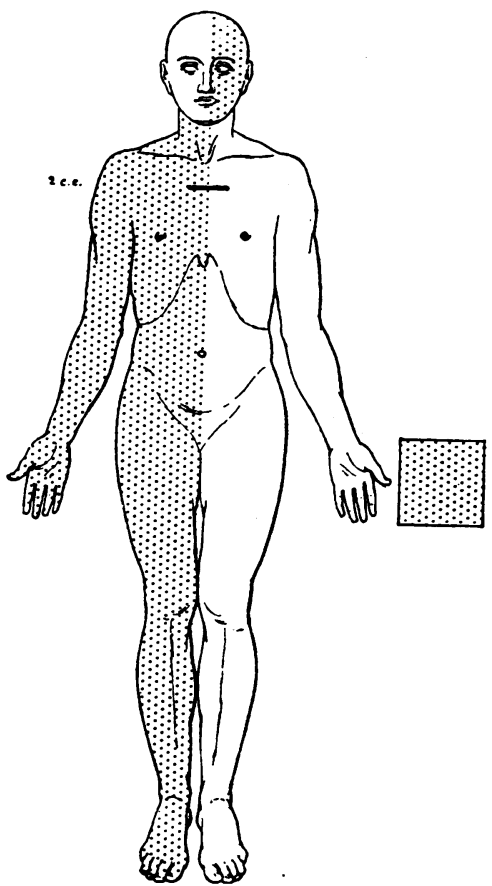

FIG. 5.-Very faint impairment of tactile sensibility.

Posteriorly there was a doubtful disturbance of sensation over the right half of the scalp.

Other Forms of Sensibility.-Power of Localization and Compass Points Test : The power of localization of a point touched could not be said to differ on the extremities of the two sides or on the two halves of the face, and was everywhere within normal limits. Compass points at $1 \mathrm{~cm}$. or even $5 \mathrm{~mm}$. distance apart were equally well recognized upon the two hands. On the face tests gave similar results upon the two sides.

Perception of Vibrations: The vibrations of a tuning fork were more distinctly felt, and lasted longer, upon the left extremities and side voL. IV.-No. 14 . 
of the body, than upon the right. The difference between the two sides was not great.

Appreciation of Difference in Weight : Several small loaded boxes of similar size, varying by $10 \mathrm{grm}$. from 10 to $100 \mathrm{grm}$. in weight, were employed in this test. Care was taken to place each box used very gently upon the patient's upturned palm. In some tests the patient was allowed to move the hand up and down two or three times; in others the hand lay upon a support. Over a series of examinations occasional mistakes were made with the patient's right hand between weights differing by $10 \mathrm{grm}$. These mistakes were chiefly made between the weights at the foot of the scale, 10 and $20 \mathrm{grm}$. The results of tests with the left hand were less faulty ; scarcely any mistakes were made.

Stereognostic Sense: The stereognostic function, as tested by the placing of various common articles in the patient's hands, seemed to be unaffected. Minor differences in texture, smoothness and roughness were, if anything, better recognized with the left than with the right hand, but the inequality in sensitiveness between the two hands was very slight.

Sense of Position in Space and of Passive Movements (Muscular Sense) : The perception of passive movements was equally good for the extremities of either side. The sense of position in space also seemed to be unimpaired.

Pressure Pain Sense: An algometer was employed so that the results might be recorded. In the application of pressure to the face, head and extremities, the part examined was always supported, so that gross displacement of the head or of a limb could not take place. When the instrument was pressed against the cheek, its point of pressure was supported by a finger placed within the mouth.

Reference to Figs. 6 and 7 will show that an appreciably greater pressure was required to produce pain upon the left side of the face and right extremities and half of the body than upon contralateral parts. This difference was most noticeable between the two hands. The numbers on the charts represent pressure in kilos and are the main results of numerous observations (Figs. 6, 7).

In the motor system the grasps and power of the two arms were equally good. The left leg was a little stronger than the right. No muscular wasting or fibrillation was anywhere observed. Muscle tone, as tested upon the two sides by clinical methods, showed no disparity from the normal. A trace of left-sided asynergia was detected in both arm and leg, which, however, subsequently disappeared. Reflexes: The tendon jerks of the right limbs were slightly more active than those of the left. The right plantar gave a doubtful response, while the left was definitely flexor in type; the superficial abdominal reflexes were present upon both sides. No involvement of the sphincters was present. 
Trophic Functions: Three deep cracks in the skin of the fingers and a blister on the palm of the right hand were present. None of these lesions had at any time given rise to pain.

Subsequent examination over a period of two months showed no change in the appreciation of the various forms of sensibility mentioned. The subjective sensation of coldness of the left face and right extremities was still complained of, but to a less degree. A slight difference in tactile sensibility between contralateral parts was present, as on the first examination.

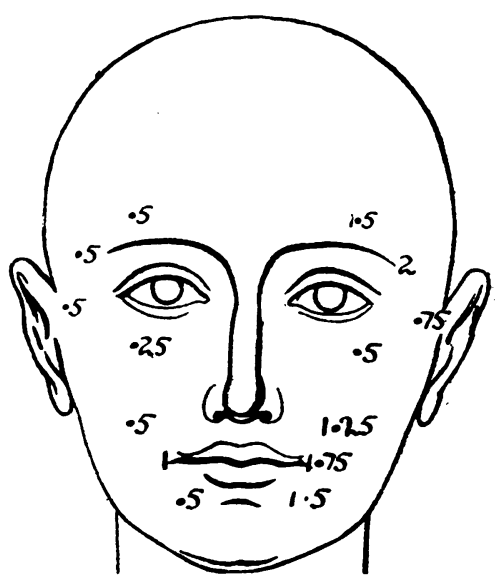

Fig. 6.-Deep pressure pain on face. The trace of asynergia observed on admission in the left extremities could not be detected subsequently. Also the muscular power of the two sides appeared to have become equalized. The right knee-jerk was still found more brisk than the left, and the right plantar continued to give an indefinite response, the left that of a purely flexor type.

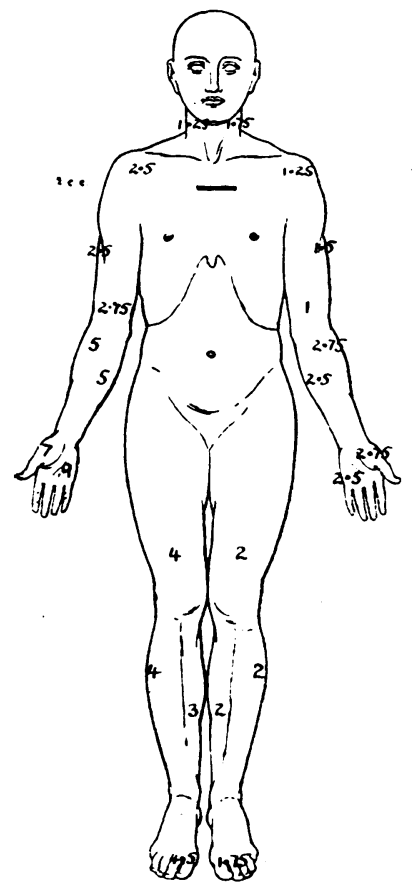

FrG. 7.-Deep pressure pain on body.

\section{SITE OF THE LESION.}

From the description of the symptoms in the above case, it is apparent that a lesion existed in the left half of the medulla. The disturbance in function of the ninth, tenth and twelfth cranial nerves and the integrity of function of the sixth, seventh and eighth nerves at a higher level, and of the eleventh below, were strongly in favour of a lesion having its greatest dimensions in the medulla at the level of the olive.

The area of damage, presumably, did not extend across the median raphé, as there was an absence of any signs of sensory impairment of the posterior column type in the left half of the body.

\section{NATURE OF THE LESION}

The age of the patient and the absence of cardio-vascular degeneration rendered improbable the existence of a vascular lesion. 
Both in the blood and C.S.F. a negative Wassermann reaction was obtained. No evidence of a tuberculous focus was discovered anywhere, and the possibility of such a lesion being present was not strengthened by the absence of a relative lymphocytosis in the blood. Further, a negative blood complement-fixation test with T.B. antigen was obtained. That the case might possibly be one of tumour was more difficult to disprove. In favour of the diagnosis of syringobulbia there was the presence of trophic changes, which were among the earliest symptoms to appear. Also the progression of symptoms was relatively slow, and no further advance in the malady occurred during the two months the patient was under observation.

\section{DISCUSSION.}

In an analysis of the symptoms produced by a unilateral lesion of the medulla, the main interest lies in the presence of disturbance in some forms of sensation and in the integrity of others. Justly it can be said that from clinical evidence alone one is restricted to indicating the dissociation of the forms of sensation, without dogmatizing upon the localization of their paths of transmission. Assumptions, however, as to the localization of the sensory tracts in the medulla are not unjustifiable when they are based upon the evidence obtained from the pathological investigations of other cases of a similar type.

In the case reported, interference with the function of the left glossopharyngeal nerve was indicated by the marked hypalgesia of the fauces, pharynx, and of the posterior third of the tongue upon the left side; light touch, on the other hand, was appreciated over the same area, but less well than upon the opposite side. There were, then, indications of a dissociated anæsthesia in the territory of the ninth cranial nerve. The left vocal cord was paralysed, and the pharyngeal and palatal reflexes were abolished, except for a very sluggish pharyngeal reflex upon the right side. Therefore the tenth cranial nerve was also affected. Cases of unilateral lesions of the medulla, verified pathologically, have been reported in which the pharyngeal and palatal reflexes were abolished upon both sides. The explanation given was the bilateral innervation of these structures.

In the territory of the fifth there was evidence that the descending root of this nerve had been damaged. The functions of the motor fifth, sixth and seventh cranial nerves were undisturbed. It is, therefore, probable that the uppermost part of the descending root of the fifth had escaped injury at the level of these structures in the lower part of the pons. In the trigeminal area upon the side of the lesion a dissociation was present of the sensations of temperature, pain and pressure from that of touch, which was disturbed to a much less degree. Further dissociation of sensation in the same area was evidenced by the fact that localiza- 
tion of a point touched and the recognition of two points simultaneously applied were correctly appreciated.

As regards tactile sensation in the trigeminal area, Bergmark ${ }^{1}$ gives it as his opinion that the sense of touch cannot be localized to the descending root alone, but chiefly to the main sensory nucleus of this nerve in the pons. In support of this view, he quotes the opinion of Brouwer, who had come to the same conclusion.

Bergmark cites two cases of medullary lesions, both anatomically investigated, one being a case of his own, and the other that of Monakow and Brun, in which the descending root of the fifth was included in the area of damage. In each case the lesion was due to occlusion of a posterior inferior cerebellar artery. Pain and temperature senses in both these cases were affected on the face upon the side of the lesion, while the sense of touch was undisturbed. In a case of Wilson's, ${ }^{2}$ also of thrombosis of a posterior inferior cerebellar artery, the lightest touch was stated by the patient to be less 'tickly' on the face on the side of the lesion, while sensibility to pain and temperature was markedly affected. Bergmark also calls attention to an unusual case of a similar nature clinically examined only, that of $\mathbf{E}$. Müller, in which the senses of touch and pressure were impaired over the first division of the trigeminus, while pain and temperature were not disturbed on the face upon the side of the lesion. Upon the opposite half of the face hypalgesia and thermal hypæsthesia were present with normal tactile appreciation.

There is considerable justification, therefore, for considering that the sense of touch in the territory of the fifth nerve is but partially represented in its descending root.

It is recognized, however, that in unilateral lesions of the medulla the sensory disturbances on the face need not be limited to the side of the lesion. Many cases have been reported in which there was disturbance of sensation upon both sides. In Wilson's case of thrombosis of the left posterior inferior cerebellar artery, the appreciation of all degrees of heat was lost upon the left face, while similar stimuli were felt as tepid upon the right half of the face. Sensibility to cold was lost upon both sides. The loss to pin-prick was upon the left side only.

In Wallenberg's ${ }^{3}$ case, of occlusion of the left posterior inferior cerebellar artery and adjacent portion of the vertebral above, pain and temperature were diminished on the right side of the face and over the first and second branches of the fifth on the side of the lesion. Tactile sensation was unimpaired.

In E. Müller's ${ }^{4}$ case 1, already mentioned, the sensory disturbance upon the face was bilateral. Tactile sensation and pressure sense were impaired over the first division of the fifth on one side, while pain and temperature were disturbed upon the opposite half of the face.

In case 3 of Gordinier, ${ }^{5}$ of thrombosis of the right posterior inferior 
cerebellar artery, he found a reduction to pain and temperature on the right side of the face and loss of the same on the left side.

Still further variations in sensation in the trigeminal areas have been discovered in cases of unilateral lesions of the medulla. The face upon the side of the lesion may have all forms of sensation intact. In Müller's ${ }^{4}$ case 2, pain and temperature were diminished on the side of the face opposite the lesion, tactile and pressure sensation being normal. Sensation over the face on the side of the lesion was unimpaired. A similar type of sensory disturbance was found by Gordinier in his case 2 .

The difficulty in understanding such variations in the sensory disturbances effected by unilateral lesions of the medulla is considerable. It must be remembered, however, that we are often not dealing with the descending root of the fifth by itself. There are also the associated cells in the substantia gelatinosa, with their more central quinto-thalamic fibres, which are liable to be damaged by lesions in this region. It is thought that these fibres arising from the cells in the substantia gelatinosa cross over to the opposite side at an early stage in their course. The direction followed by these fibres in traversing the median raphe is uncertain. Wallenberg suggests that they take a dorso-medial direction passing ventral to the position of the twelfth nucleus, decussate and turn upwards in the lateral region of the formatio reticularis. Bury and Stopford ${ }^{6}$ diagrammatically represent the crossed position of these fibres in the medulla as lying dorso-medially and in close apposition to the spino-thalamic tract.

Such an arrangement, though not conclusively proved, at any rate makes it more casy to understand how sensory disturbances may be present on one or both sides of the face in cases of unilateral lesions of the medulla. For it is obvious that the sensory disturbances produced by such a lesion must vary in accordance with the number of these structures mentioned above which have been damaged.

Thus, Bury and Stopford describe a case of occlusion of a posterior inferior cerebellar artery in which the sensibility to pain and temperature was disturbed upon the face, extremities and trunk on the side opposite to the lesion in the medulla. In this case they postulate that the crossed fibres subserving pain and temperature for the whole of one half of the body in the spinothalamic and quinto-thalamic tracts had been damaged in their course through the medulla, and that the descending root of the fifth had not been disturbed by the lesion. The absence of damage to the descending root of the fifth on the side of the lesion would account for the integrity of sensation upon the face on the same side.

An additional point of interest in connection with the sensory disturbances in the territory of the fifth nerve is met with sufficiently often to be worthy of attention. Frequently the impairment, in the sensations of pain and temperature especially, is found to vary in depth 
over different areas of the trigeminal distribution. The disturbance is usually greatest over the forehead, and less within the territory of the second and third divisions. In the case described in this paper the reduction in the thermal and painful sensibility was greatest in the area of the first division. The area of distribution of the second was less, and of the third least, affected. In both of E. Müller's cases, in which there was hypalgesia and thermal hypæsthesia over the whole of the opposite half of the body, including the face, from the side of the lesion, the impairment in the trigeminal area was greatest in the region of the first branch.

By way of contrast to the above, case 1 of Breuer and Marburg 7 may be mentioned, in which the disturbance of pain sensibility on the face on the same side as the lesion was more profound in the third division of the fifth, although all three divisions were affected. A pathological examination in this case revealed the presence of thrombosis of the left vertebral artery. The area of damage in the medulla was the same as that commonly found to result from occlusion of a posterior inferior cerebellar artery.

An inequality in the depth of sensory disturbance in the divisional areas of the trigeminal nerve, at any rate when present upon the same side as the lesion, may be accounted for in the following manner. It is probable that a nuclear systematization exists of the sensory fibres of the trigeminus, which pass into its descending or spinal root. Thus, from below upwards the terminations of the ophthalmic, superior maxillary and inferior maxillary fibres which enter the descending root are considered to be in that order. Among those authors who give it as their opinion that such an arrangement exists are Wallenberg, Bergmann and Schlesinger. It is obvious that a lesion in the medulla falling short of the main sensory nucleus of the fifth nerve in the pons will leave undisturbed a varying proportion of the descending root in its uppermost part. One would expect, therefore, the sensory disturbance in the third division to be more often of less intensity than in the second or first divisions.

In opposition to the above localization there is the theory of Woods, ${ }^{8}$ who describes two cases in which the disturbance of pain and temperature was suggestive of a segmental distribution. He considers that there is a representation in the descending root of successive cutaneous zones, the outermost of which is limited by a line from the vertex of the skull by way of the ear to the chin, each succeeding zone being of smaller radius until the nose is reached. Representation for the outermost zone he assigns to the caudal end of the descending root of the fifth, and so on. His first case was one of syringomyelia and syringobulbia. In many cases of such a nature the outline of the area of impaired sensibility upon the face not infrequently corresponds to one or other of the zones 


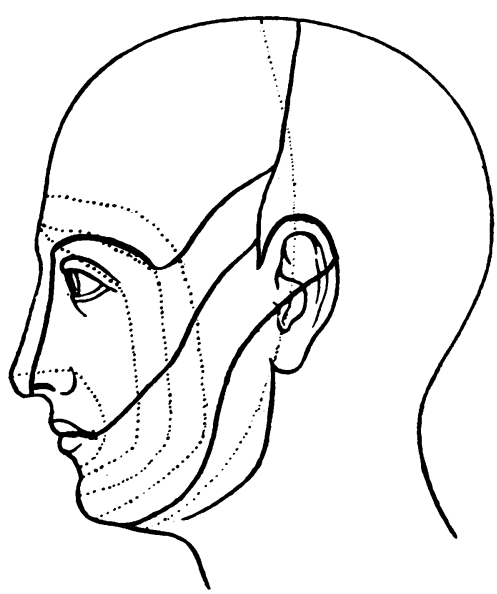

FIG. 8.-Composite diagram. The continuous lines indicate the areas supplied by the trigeminal branches. The dotted lines indicate possible segmental areas of the quinto-thalamic fibres.

described above. It is suggested that such sensory changes are due not to disturbance of the descending roots so much as to interference with the crossed or uncrossed portions of the quinto-thalamic fibres as they traverse the medulla, while at the same time undergoing a regrouping of a segmental character (Fig. 8).

A considerable disturbance in the sense of pressure pain was present on the face in the case described in this paper upon the same side as the impairment in the sensations of pain and temperature. This coincides with the deduction of Head and Thomson, ${ }^{9}$ that there is loss to both deep and superficial painful stimuli when the lesion is above the peripheral level.

G. W. Robinson ${ }^{10}$ agrees that a lesion of the spinothalamic tract in the cord makes no distinction between pain of a deep or superficial quality, and that they are also closely associated in the brain stem. He states, however, that on the face, while superficial pain may be grossly affected, the algometer may indicate only a partial loss of deep pain sense. From their researches upon the sense of pressure pain on the face, eye and tongue, Maloney and Foster Kennedy ${ }^{11}$ conclude that in intracranial interference with the fifth nerve the sense of pressure pain is disturbed.

On the other hand, in Wilson's case the sense of pressure pain was unaffected upon the side of the face presenting reduction in both painful and thermal sensibility. In a case reported by Robinson, ${ }^{\mathbf{1 0}}$ pressure pain was not disturbed on either side of the face, but was reduced on the extremities and trunk contralaterally to the lesion in the medulla. Superficial pain was impaired over the opposite side of the face to the lesion.

It is, therefore, apparent that the association of the superficial and deep forms of pain from the trigeminal area is capable of disruption within the medulla.

In regard to the sense of taste, this form of sensation was found to be intact upon both sides of the tongue in the case described. Gustatory sensations, therefore, were dissociated on the left half of the tongue from those of pain, painful pressure, and, to a less degree, from touch.

Considerable dubiety still exists in connection with the question whether any of the fibres subserving the function of taste enter the 
brain stem in the fifth nerve or not. It is generally held that the bulk of the gustatory fibres from the anterior two-thirds of the tongue ascend by the lingual, chorda tympani and seventh nerves to the geniculate ganglion, and enter the brain stem by the nervus intermedius of Wrisberg.

After entering the brain stem, in close relationship with the seventh and eighth nerves, the nerve of Wrisberg traverses the dorsal part of the descending root of the fifth at a high level. The fibres then bend downwards to enter the tractus solitarius, which in a cross section of the medulla lies just ventrolaterally to the ala cinerea of the floor of the fourth ventricle. In the nucleus of this tract the fibres terminate.

The nucleus associated with the tractus solitarius extends throughout the entire length of the medulla and receives the fibres of the nervus intermedius and the gustatory fibres of the glossopharyngeal nerve (Ranson). It is probable in cases of unilateral lesions of the medulla that the state of integrity of the sense of taste on the side of the lesion depends entirely upon the presence or absence of damage to the above tract and its nucleus.

According to Nageotte, ${ }^{12}$ on the other hand, the gustatory nucleus extends upwards into the pons, and is at its superior extremity in intimate relationship with the sensory nucleus of the fifth. At its lower end in the medulla it is in contact with the uppermost part of the tractus solitarius, constituting what is known as the nucleus or gelatinous substance of this tract. Nageotte is of the opinion that trigeminal fibres subserving taste enter the gustatory nucleus at its upper end. The entry zone for the fibres of the nervus intermedius he considers to be in the middle portion of the nucleus, the taste fibres from the glossopharyngeal nerve entering at a lower level. In support of his argument he quotes an observation of Wallenberg's on a case which showed anæsthesia in the left trigeminal region, loss of taste at the back of the tongue on the left side, and left-sided hemiatrophy of the tongue. The hypoglossal and part of the trigeminus were damaged by the presence of a tumour, while the nerve of Wrisberg and glossopharyngeal nerve were intact. Degeneration of the fibres attached to the gustatory nucleus was greatest at its upper end. For this reason, Wallenberg came to the conclusion that taste fibres were present in the fifth nerve.

Cushing, ${ }^{13}$ however, in a series of cases found that the sense of taste remained unaffected after the removal of the Gasserian ganglion. It may, therefore, be inferred that, if any taste fibres exist in the trigeminal nerve, the severance of these fibres does not normally produce any demonstrable impairment in the sense of taste.

Raymond and Français ${ }^{15}$ describe a case of a unilateral pontine lesion in which there was absence of taste on the anterior two-thirds of the tongue, while sensation in the territory of the fifth upon the same side was unaffected. They remark that in this case it is difficult to 
suppose that the fifth nerve played any part at all in the perception of gustatory impressions.

Concerning taste upon the posterior third of the tongue, Henschen ${ }^{14}$ reports a case of a unilateral lesion of the medulla in which there was, at the first examination, normal taste sense over the posterior third of the tongue on the side of the lesion. On the anterior two-thirds of the same side taste was impaired. At a later date the sense of taste over the posterior third became 'lighter' and delayed. In the majority of cases, however, of unilateral lesions of the medulla, it is usual to find the sense of taste either preserved or disturbed upon the whole half of the tongue.

The remote effects of the lesion in the case described in this paper consisted of a crossed disturbance of painful, thermal and pressure pain sensations upon the extremities and half of the trunk; tactile sensation was dissociated, being only lightly disturbed in comparison to these other forms of sensation. From the raising of the thresholds of pain and painful pressure in the parts mentioned, additional evidence is gained in support of the view that their paths of transmission are closely associated as they pass through the medulla in the spinothalamic tract.

Disturbance in the power of localization, appreciation of two compass points and of weights, stereognosis, vibration and muscular sense was restricted to a very slight impairment in a limited number of these forms of sensibility. These forms of sensation are commonly supposed for the most part to have their pathway in the lemniscus at the level under discussion. That the sense of vibration, appreciation of weights, and stereognosis, showed slight impairment in the right extremities suggested some interference with the lemniscus on the side of the lesion.

The integrity of the muscular sense is of interest. Bergmark considers that this form of sensibility must have a double pathway, one being in the posterior columns and the lemniscus, and the other in the direct cerebellar tract of Flechsig. Cases are on record of unilateral pontine lesions at the level of the decussation of the superior cerebellar peduncles, in which the muscular sense was severely affected upon the contralateral side of the body. Presumably in such cases decussation had occurred of both possible pathways of muscular sense. In the bulb, a lesion affecting either Flechsig's tract or the lemniscus in the opposite half of the cord has been found in several cases to have produced in the acute stages some disturbance of the muscular sense, which has later disappeared. The recovery of the muscular sense was due apparently to the fact that one of its possible paths of transmission remained undisturbed.

Bergmark describes a case of tumour at the level of the foramen magnum which produced severe compression and demyelinization of the 
posterior columns and of Flechsig's tract upon one side. In this case the muscular sense on the same side was greatly disturbed.

\section{REFERENCES.}

1 G. Bergmark, Upsala Lakaref. Förh., 1911, xxvi, H. 5-6.

2 S. A. K. Wilson, Proc. Roy. Soc. Med. Lond., February, 1909.

3 Wallenberg, Arch. f. Psychiat., 1895, xxvii, 504.

4 E. Müller, Deut. Zeits. f. Nervenh., 1906, xxxi, 452.

5 H. C. Gordinier, Albany Med. Annals, October, 1911.

- Bury and Stopford, Med. Chronicle, December, 1913.

7 Breuer and Marburg, Obersteiner's Arbeiten, 1902, ix, 181.

8 Woods, Journ. Nerv. Ment. Dis., 1913, xl, 91.

9 HeAD and Thomson, Brain, 1906, xxix, 537.

10 G. W. Robinson, Jour. Amer. Med. Assn., 1913, 1xi, 179.

11 Maloney and Foster Kennedy, Brain, 1911, xxxiv, 1.

12 NAgeotTf, Review Neur. and Psychiat., 1906, iv, 473.

13 Cushing, Johns Hopkins Hosp. Bull., 1904, xv, 213.

14 Henschen, Neur. Centralb., 1906, xxv, 502.

15 RAymond and FraxçaIs, Revue neurol., 1909, xvii, 445. 